\title{
Fabricación de tableros enlistonados con madera proveniente del manejo de renovales de raulí
}

\author{
Blockboards manufacture with rauli wood from thinnings of second growth forests
}

C.D.O.: 832.286

H. POBLETE, J.E. DIAZ-VAZ, R. JUACIDA, P. NIEMZ, A. ROLLERI

Instituto de Tecnología de Productos Forestales, Universidad A ustral de Chile, Casilla 567, Valdivia, Chile.

\section{SUMMARY}

Second growth Raulí (Nothofagus alpina) thinnings and Pinus radiata wood was used as raw material for the manufacturing of blockboards. As adhesive polivinyl acetate was applied. Wood yield on blockboard production and tensile strength of wood and lap joints were studied.

$Y$ ield of $58 \%$ was determined for the production of blockboards with dryed $N$. alpina saw wood. This value was higher than those reported for $P$. radiata by a furniture industry (35-40\%).

Tensile strength of $N$. alpina was about $4 \mathrm{~N}$, almost $40 \%$, higher than $P$. radiata. There were no differences between Raulí splint and heart wood. Tensile strength of lap joints of $N$. alpina were superior to those required by EN 204 Standard. After air conditioning a tensile strength of about $12 \mathrm{~N}$ with $N$. alpina and $9 \mathrm{~N}$ with $P$. radiata was determined. Soaking in water for 96 hours at $20^{\circ} \mathrm{C}$ reduced values to $5.5 \mathrm{~N}(N$. alpina) and $6.0 \mathrm{~N}(P$. radiata).

\section{RESUMEN}

Se fabricaron tableros enlistonados, "blockboards", con madera proveniente del raleo de renovales de Raulí (Nothofagus alpina) y Pino Insigne (Pinus radiata) de plantaciones industriales. Como adhesivo se usó polivinil acetato. Se determinó el rendimiento y la resistencia al cizalle de la madera y de las uniones.

El rendimiento de $N$. alpina al producir tableros desde madera aserrada seca fue de $58 \%$, valor superior al reportado por la industria del mueble con $P$. radiata (35-40\%).

$L$ a resistencia al cizalle de la madera de Raulí fue alrededor de $4 \mathrm{~N}(40 \%)$ más alta que la de P. Insigne. No se registraron diferencias entre albura y duramen de Raulí. Las resistencias al cizalle de las uniones de Raulí superaron las exigencias de la norma EN 204. Luego de un climatizado la resistencia de $N$. alpina fue de $12 \mathrm{~N}$, mientras que con $P$. radiata sólo se alcanzaron $9 \mathrm{~N}$. Un tratamiento en agua a $20^{\circ} \mathrm{C}$ por 96 horas redujo esta propiedad a 5.5 . N en $N$. alpina y 6.0 en $P$. radiata.

\section{INTRODUCCION}

El presente estudio forma parte de una serie de ensayos que pretende determinar la aptitud tecnológica de la madera proveniente del manejo de renovales de Raulí (Nothofagus alpina) para la fabricación de productos industriales. EI trabajo es parte de los resultados de un Proyecto FONTEC patrocinado por Forestal Mininco S.A. y Forestal Río Vergara S.A. (Poblete, Díaz-Vaz, Juacida, 1994).

Se determina la aptitud de esta madera para la fabricación de tableros enlistonados tipo "blockboard", con uniones en el sentido Iongitudinal de las fibras. La fabricación de este tipo de tableros da un mayor valor agregado a una materia prima que actualmente encuentra mercado sólo en las astillas de exportación.

En el estudio de la calidad de este producto resulta fundamental el lograr una buena unión entre las piezas de madera. La calidad del producto depende tanto del adhesivo como de la resistencia de la especie de madera empleada (Poblete, 1992a, b). 


\section{MATERIAL Y METODO}

Para la fabricación de los tableros enlistonados, "blockboards", se empleó una muestra de madera aserrada seca proveniente del aserrado de trozas obtenidas del raleo de renovales existentes en la IX Región (Nahuelbuta, Jauja y Pichibureo). Se incluyó una muestra de tableros confeccionados con Pinus radiata a modo de testigo.

Durante el proceso de fabricación se realizó un estudio de rendimiento. Para ello se consideró el volumen de madera aserrada seca ingresado al proceso y el volumen de madera obtenido luego del precortado y cepillado requerido para la confección de los tableros. En el estudio del rendimiento se emplearon 290 tablas provenientes de las zonas de muestreo señaladas.

Como adhesivo se utilizó polivinil acetato formulado para cumplir con las condiciones de uso en exteriores, protegido de las inclemencias climáticas.

El proceso de fabricación de tableros se llevó a cabo en una prensa industrial discontinua con condiciones de tiempo, presión y temperatura dadas por una industria de muebles (INTERVAL Ltda.).

Los ensayos se realizaron de acuerdo con las normas europeas EN 204, "Evaluación de adhesivos para unir maderas y productos derivados de madera" y EN 205, "Determinación de la adhesión de uniones longitudinales por cizalle".

Tomando en consideración las características del adhesivo a emplear en el presente estudio y al uso estimado de los paneles (muebles de interiores), se aplicó el método de evaluación para la clase D-3.

A parte de ensayar las uniones de madera con adhesivo, para comprobar la resistencia de la made$\mathrm{ra}$, se confeccionaron probetas gemelas de madera sólida, sin unión. Al cortar las probetas se separaron en albura, duramen y unión de albura con duramen.

Las exigencias de resistencia para los distintos tratamientos de envejecimiento y una descripción de éstos se presenta en el cuadro 1.

La forma de las probetas correspondió a la estipulada por la norma y se presenta en la figura 1.

Las probetas se confeccionaron con la dirección de las fibras paralelas al eje mayor de la probeta. El corte transversal (ranura) se realizó con precisión hasta la línea de cola. Las probetas no presentaban nudos u otros defectos.

En el caso del ensayo, para comprobar la resistencia de la madera, las probetas se confeccionaron como las descritas anteriormente pero sin incluir en ellas una unión con adhesivo. Estas
CUADRO 1

Tipos de tratamiento y resistencia mínima exigida por EN 204 Clase D-3.

Test conditioning and strength requirements of EN 204 Standard for Boardclass D-3.

\begin{tabular}{|c|c|c|}
\hline $\begin{array}{l}\text { Exigencia } \\
\text { Norma EN } \\
\mathrm{N} / \mathrm{mm}^{2}\end{array}$ & $\begin{array}{l}\text { Tratamiento } \\
\mathrm{N}^{\circ}\end{array}$ & Características \\
\hline$>10$ & 1 & 7 días climatizado DIN 50014 \\
\hline$>2$ & 2 & $\begin{array}{l}7 \text { días climatizado DIN } 50014 \\
4 \text { días agua fría }\end{array}$ \\
\hline$>6$ & 3 & $\begin{array}{l}7 \text { días climatizado DIN } 50014 \\
4 \text { días agua fría } \\
7 \text { días climatizado DIN } 50014\end{array}$ \\
\hline
\end{tabular}

probetas "sin unión" se sometieron a los mismos tratamientos de envejecimiento que considera la norma. Las dimensiones de estas probetas fueron exactamente iguales a las con unión. El aspecto de la probeta sin unión se muestra en la figura 1.

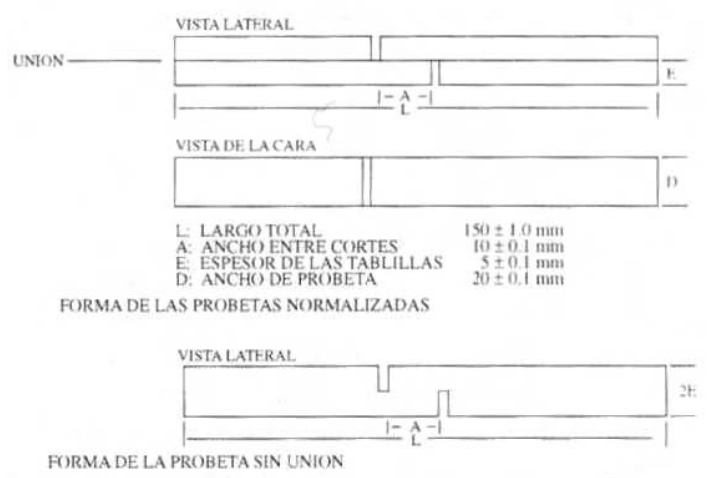

Figura 1. Forma de las probetas.

Test pieces.

\section{RESULTADOS}

3.1. Estudio de rendimiento. EI volumen de la muestra de madera aserrada seca ingresada al proceso fue de $2.64 \mathrm{~m}^{3}$, el volumen procesado que se obtuvo fue de $1.55 \mathrm{~m}^{3}$, con lo que se determinó un rendimiento general de $58.6 \%$.

En el cuadro 2 se presenta el detalle de la cantidad y dimensiones de las piezas obtenidas por zona de muestreo.

En el proceso de aserrío se obtuvo un rendimiento promedio, desde trozo a madera aserrada, de $43.2 \%$. Al transformar la madera aserrada en 


\section{CUADRO 2}

Rendimiento de la madera procesada. Espesor de las piezas: $22 \mathrm{~mm}$.

Wood yield. End board thickness $22 \mathrm{~mm}$.

\begin{tabular}{|c|c|c|c|c|c|c|}
\hline \multirow{2}{*}{$\begin{array}{l}\text { Zona de } \\
\text { muestreo }\end{array}$} & \multirow{2}{*}{$\begin{array}{c}\text { Volumen } \\
\text { ingresado } \\
\mathrm{m}^{3}\end{array}$} & \multicolumn{3}{|c|}{ Piezas obtenidas } & \multirow{2}{*}{$\begin{array}{c}\text { Volumen } \\
\text { obtenido } \\
\mathrm{m}^{3}\end{array}$} & \multirow{2}{*}{$\begin{array}{c}\text { Rendimiento } \\
\%\end{array}$} \\
\hline & & $\begin{array}{c}\text { Ancho } \\
\mathrm{mm}\end{array}$ & $\begin{array}{c}\text { Largo } \\
\mathrm{mm}\end{array}$ & $\begin{array}{c}\text { Cantidad } \\
\mathrm{N}^{\circ}\end{array}$ & & \\
\hline \multirow[t]{7}{*}{ Pichibureo } & & 60 & 1.000 & 161 & 0.212 & \\
\hline & & 75 & 750 & 71 & 0.088 & \\
\hline & & 90 & 520 & 77 & 0.079 & \\
\hline & & 75 & 520 & 54 & 0.046 & \\
\hline & & 80 & 520 & 35 & 0.032 & \\
\hline & & 75 & 420 & 60 & 0.042 & \\
\hline & 0.90 & & & & 0.499 & 55.44 \\
\hline \multirow[t]{7}{*}{ Jauja } & & 65 & 1.000 & 250 & 0.358 & \\
\hline & & 75 & 520 & 13 & 0.011 & \\
\hline & & 90 & 520 & 22 & 0.022 & \\
\hline & & 75 & 420 & 37 & 0.025 & \\
\hline & & 90 & 420 & 8 & 0.007 & \\
\hline & & 75 & 750 & 235 & 0.291 & \\
\hline & 1.19 & & & & 0.714 & 60.00 \\
\hline \multirow[t]{6}{*}{ Nahuelbuta } & & 65 & 1.000 & 132 & 0.189 & \\
\hline & & 90 & 420 & 19 & 0.016 & \\
\hline & & 75 & 420 & 6 & 0.004 & \\
\hline & & 90 & 520 & 7 & 0.007 & \\
\hline & & 75 & 750 & 95 & 0.118 & \\
\hline & 0.55 & & & & 0.333 & 60.73 \\
\hline
\end{tabular}

tableros se registró un rendimiento promedio de $58.6 \%$. Con estos valores se pudo determinar que el rendimiento promedio de troza a tablero enlistonado fue de $25.3 \%$ (cuadro 3 ).

\section{CUADRO 3}

Rendimiento de la madera procedente del manejo de renovales en la fabricación de tableros enlistonados.

Y ield of second growth Raulí wood with manufactured blockboards.

\begin{tabular}{|lccc|}
\hline \multirow{2}{*}{$\begin{array}{l}\text { Zona de } \\
\text { muestreo }\end{array}$} & \multicolumn{3}{c|}{ Rendimientos (\%) } \\
\cline { 2 - 4 } & $\begin{array}{c}\text { De trozo } \\
\text { a aserrado }\end{array}$ & $\begin{array}{c}\text { De aserrado } \\
\text { a tableros }\end{array}$ & $\begin{array}{c}\text { De trozo } \\
\text { a tableros }\end{array}$ \\
\hline Pichibureo & 43.6 & 55.4 & 24.2 \\
Jauja & 39.7 & 60.0 & 23.8 \\
Nahuelbuta & 46.2 & 60.7 & 28.0 \\
\hline Promedio & 43.2 & 58.6 & 25.3 \\
\hline
\end{tabular}

Los resultados obtenidos en rendimiento con la madera proveniente de raleos de Raulí son alentadores. De acuerdo con la información obtenida en la industria (INTERVAL S.A.), los rendimientos obtenidos con Pino insigne, de madera aserrada a tablero, son más bajos y fluctúan entre 35 y $40 \%$, debido a la eliminación de nudos y otros defectos. 3.2. Propiedades de los tableros. Como se mencionara anteriormente, a modo de control de las propiedades de la madera, se fabricaron probetas de cizalle en las que no se incluía una unión con adhesivo. Estas probetas se sometieron a los tratamientos que indica la norma y se ensayaron determinándose la resistencia al cizalle.

Los resultados de este ensayo se presentan en la figura 2. En el ensayo se diferenció entre madera de duramen y de albura. Se incluyeron, a modo de testigo, probetas de Pinus radiata.

La resistencia de la madera de renovales es notoriamente superior a las exigencias de la norma (cuadro 1). Este hecho permite asegurar que si 
Cizalle $\left(\mathrm{N} / \mathrm{mm}^{2}\right)$

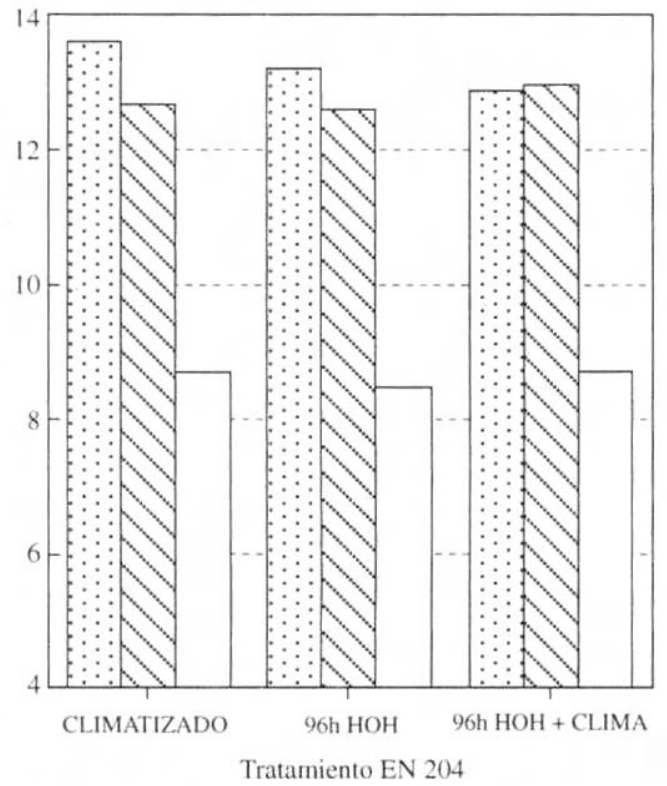

Albura $\bigcirc$ Duramen $\square$ Pino insigne

Figura 2. Resistencia de la madera al ensayo de cizalle (Probetas sin unión) según norma EN 204.

Tensile strength of wood. EN 204 Standard.

en el ensayo de las uniones se tienen resistencias inferiores a las exigidas por la norma, esta deficiencia se deberá al adhesivo o a la calidad del fraguado pero no a la madera.

Importantes resultan las diferencias de resistencia entre el material de los renovales y la madera del testigo. Con la madera de renovales se pueden fabricar tableros enlistonados que con seguridad cumplirán con los requisitos de la norma europea, mientras que con el Pino Insigne existe el peligro de que la madera no cumpla con el requisito.

El análisis de los valores obtenidos reveló que no existen diferencias significativas entre la madera de albura y la de duramen. Entre otros, esto se debería a que la madera identificada como duramen presenta una coloración rosada típica del duramen, pero probablemente se trata de un proceso de duraminización incipiente, donde no existe una diferenciación tecnológica de ambos tipos de madera.

En la figura 3 se presentan los valores promedio de cizalle de las uniones luego de los tratamientos.

Al comparar las propiedades de la madera (figura 2) con las de las uniones (figura 3), se aprecia que con los tratamientos de envejecimiento

\section{Cizalle $\left(\mathrm{N} / \mathrm{mm}^{2}\right)$}

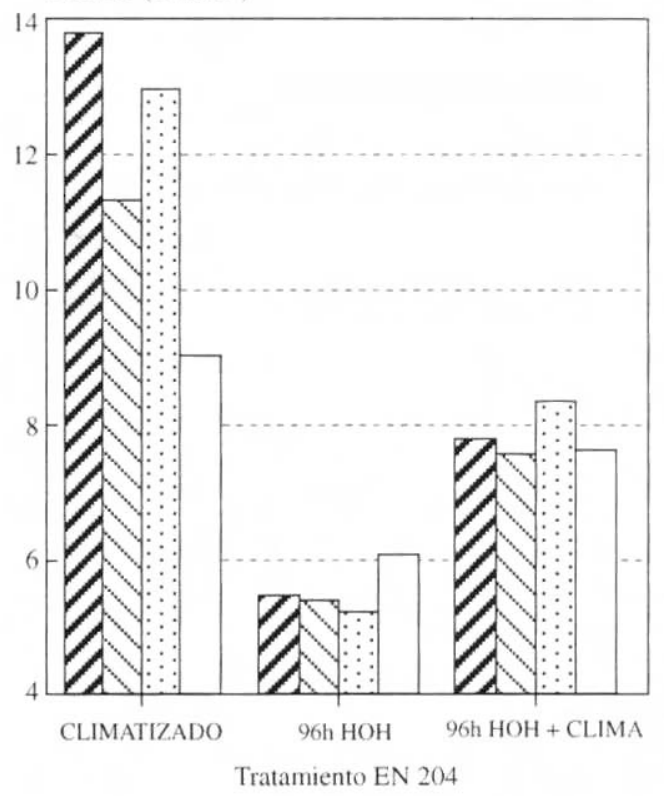

$\triangle$ Albura $\triangle$ Duramen $\quad \because$ Combinada $\square$ Pino insigne

Figura 3. Resistencia al cizalle de uniones en tableros enlistonados según norma EN 204.

Tensile strength of lap joints. EN 204 Standard.

acelerado las uniones pierden resistencia, mientras que la madera no se ve afectada por estos tratamientos.

Los resultados presentados en la figura 3 deben evaluarse comparándolos con las exigencias de la norma (cuadro 1). Los tableros confeccionados con madera de renovales superan ampliamente las exigencias de la norma en todos los tratamientos. Si se tiene en cuenta la resistencia de los testigos, elaborados con Pino Insigne, se observa que las uniones producidas con renovales dan mejores resultados, salvo en el caso del tratamiento en agua sin climatizado.

Debe destacarse que los tableros confeccionados con el testigo no cumplen con la norma en el primer tratamiento (7 días de climatizado).

La observación de las uniones de renovales indica que no existen problemas de adhesividad y que todas las fracturas provocadas por el ensayo se producen en la madera. En general, los resultados indican que la madera proveniente del manejo de renovales es una excelente materia prima para el tipo de tableros enlistonados ("blockboards") que supera a la madera de uso tradicional en este rubro, el Pinus radiata. 


\section{BIBLIOGRAFIA}

EUROPEAN COMMITTEE FOR STANDARDIZATION. 1991. Evaluation of non-structural adhesives for joining of wood and derived timber products. European Standard. EN 204. $3 \mathrm{pp}$

EUROPEAN COMMITTEE FOR STANDARDIZATION. 1991. Test methods for wood adhesives for nonstructural applications; determination of tensile strength of lap joints. European Standard. EN 205, 5 pp.

POBLETE, H. 1992a. "A varices sobre el cizalle en uniones de
Pinus radiata D. Don". Ciencia e Investigación Forestal, 6(1): 117-123.

POBLETE, H. 1992b. Calidad de las uniones en blockboards de Pinus radiata. II Simposio Pinus radiata investigación en Chile, pp. 273-284.

POBLETE H., J.E. DIAZ-VAZ,R. JUACIDA. 1994. A provechamiento industrial de la madera proveniente del manejo de renovales. Fondo Nacional de Desarrollo Tecnológico y Productivo. FONTEC-CORFO. Forestal Río Vergara S.A.; Forestal Mininco S.A., Universidad Austral de Chile Fac. Cs. Forestales. Informe de Convenio $N^{\circ} 215.215$ pp. 